\title{
Wirtschaftliche Stimmung der Ärzte bleibt düster
}

— Niedergelassene Ärzte in Deutschland bewerten ihre wirtschaftliche Lage und die Perspektiven für das erste Halbjahr 2012 eher ungünstig. Das ergibt der Medizinklimaindex (MKI) Herbst 2011 der Stiftung Gesundheit mit einem Wert von -14,9. Der MKI wird seit 2006 zweimal jährlich bestimmt. Seit der ersten Erhebung waren die Werte durchgehend negativ. Ein Rekordtief $(-36,7)$ wurde im Frühjahr 2009 errechnet.
Im Vergleich zum Frühjahr 2011 hat sich die Stimmung unter den Niedergelassenen weiter eingetrübt. Rund $38 \%$ der befragten Mediziner erwarten eine Verschlechterung ihrer wirtschaftlichen Lage, mit einer Verbesserung rechnen hingegen nur 5\%. Etwa 57\% vermuten eine gleichbleibende Wirtschaftslage.

Deutliche Unterschiede bestehen zwischen den diversen Fachgruppen: Mit -21,5 am schlechtesten ist der MKI der Humanmediziner. Die Zahnärzte zeigten sich mit einem Wert von -3,3 deutlich optimistischer. Am rosigsten sehen die Psychologischen Psychotherapeuten ihre nahe wirtschaftliche $\mathrm{Zu}$ kunft. Ihr MKI liegt bei $+4,2$.

Interessierte können die gesamte MKIAnalyse im Internet nachlesen unter www. stiftung-gesundheit.de/forschung/studien. $\mathrm{htm}$ Brigitta Schneider

\section{Yoga und Stretching lohnen sich Rückenschmerzen wegdehnen}

— Egal ob mit Yoga oder Stretching: Regelmäßiges Dehnen lindert einer aktuellen USStudie zufolge Rückenschmerzen (Arch Intern Med 2011; doi:10.1001/archinternmed.2011.524). Insgesamt 228 Rückenschmerzpatienten wurden jeweils drei Monate einmal pro Woche entweder 75 Minuten in Yoga oder 52 Minuten in Stretchingtechniken unterrichtet. In den Kursen wurden die Patienten zudem angehalten, an möglichst vielen Tagen selbständig zu üben. 63\% der Yoga- und
$82 \%$ der Stretching-Schüler schafften dies auch mindestens dreimal pro Woche. Als Kontrollgruppe fungierten Patienten, die lediglich ein Buch mit allgemeinen Informationen zu Rückenschmerzen und Vorschlägen für Übungen und Lebensstiländerungen erhalten hatten.

Den Funktionsstatus des Rückens eruierten die Mediziner mit dem Modified Roland Disability Questionnaire (RDQ) anhand einer 23-Punkte-Skala. Den Schmerzpegel maßen

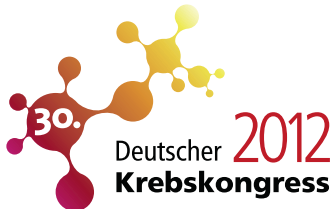

— Neben der Präsentation herausragender wissenschaftlicher Beiträge konzentriert sich der DKK 2012 auf gesundheitspolitische Themen. Im Rahmen der DKK-Veranstaltungen zum Nationalen Krebsplan werden Aspekte zur Krebsfrüherkennung, Qualitätssicherung, Arzneimittelbewertung und Patienteninformation diskutiert.

Mit der Planarsitzung „Palliativmedizin" steht bereits am ersten Tag ein Thema auf der Agenda, das jeden in der Onkologie tätigen angeht - über alle onkologischen Disziplinen hinweg. Zwei Plenarsitzungen, eine KeyNote-Lecture und eine Pro- und ContraDiskussion widmen sich verschiedenen Aspekte der palliativmedizinischen Versorgung. So diskutiert Giovanni Maio, Freiburg, in der Key-Note-Lecture das Thema „Ökonomie versus Ethik im onkologischen Behandlungsalltag" und Jutta Hübner, Frankfurt, fragt in der Plenarsitzung Palliativmedizin I nach der "Ärztlichen Kompetenz am Lebensende". Merken Sie sich Mittwoch, den 22. Februar 2012 als den Palliativtag auf dem DKK vor. Details finden Sie im Vorprogramm.

Das DKK-Vorprogramm kann ab sofort heruntergeladen werden: www.krebsgesellschaft.de/dkk_2012_programm.

Weitere Auskunft:

Kongress- und Kulturmanagment $\mathrm{GmbH}$

Postfach 3664

99407 Weimar

dkk2012@kukm.de

www.dkk2012.de

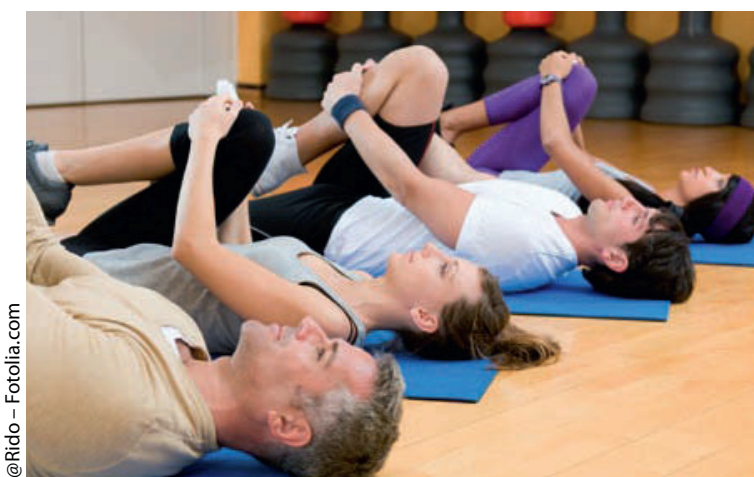

Wichtig ist, dass Rückenschmerzpatienten für sie geeignete Dehnübungen erlernen.

sie mit einer numerischen 11-Punkte-Skala. Die Daten wurden bei allen Patienten zu Beginn der Studie, nach sechs, zwölf und 26 Wochen per Telefon erhoben.

Der Schmerzmittelverbrauch reduzierte sich in der Yoga- wie auch in der Stretching-Gruppe im Verlauf deutlich, nicht jedoch in der Kontrollgruppe. Die rückenschmerzbedingten Funktionsstörungen gingen in allen drei Gruppen zurück, allerdings in deutlich unterschiedlichem Ausmaß. Im Vergleich mit der Kontrollgruppe war die Funktion bei den Yoga-Schülern und auch in der Stretching-Gruppe nach 12 und nach 26 Wochen deutlicher gebessert (mittlere RDQ-Differenz 2,5 und 1,8 Punkte respektive 2,2 und 1,5 Punkte). Am Studienende bezeichneten gegenüber der Kontrollgruppe 2,75-mal mehr Yoga- und 2,58-mal mehr Stretching-Patienten ihre Rückenschmerzen als besser, viel besser oder vollständig verschwunden. Die Autoren betonen: Da sich Yoga-Kurse in ihrer Art enorm unterscheiden, sollten Rückenschmerzpatienten bevorzugt geeigneten Kurse mit therapeutischer Orientierung zugewiesen werden. Frank Stebner 